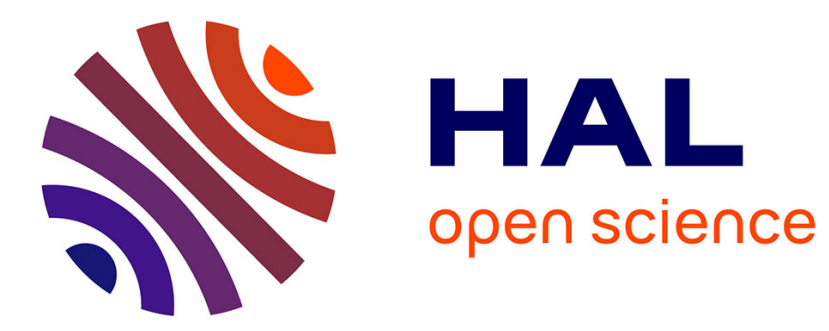

\title{
La peinture à Taiwan des années 1930 aux années 1950 : échos artistiques du contexte socio-politique
}

\author{
Marie Laureillard
}

\section{To cite this version:}

Marie Laureillard. La peinture à Taiwan des années 1930 aux années 1950: échos artistiques du contexte socio-politique. Monde chinois nouvelle Asie, 2019, 58, pp.70-82. halshs-02281162

\section{HAL Id: halshs-02281162 \\ https://shs.hal.science/halshs-02281162}

Submitted on 15 Dec 2020

HAL is a multi-disciplinary open access archive for the deposit and dissemination of scientific research documents, whether they are published or not. The documents may come from teaching and research institutions in France or abroad, or from public or private research centers.
L'archive ouverte pluridisciplinaire HAL, est destinée au dépôt et à la diffusion de documents scientifiques de niveau recherche, publiés ou non, émanant des établissements d'enseignement et de recherche français ou étrangers, des laboratoires publics ou privés. 


\section{MONDE CHINOIS nouvelle Asie

\section{Directeur de la publication}

Serge KEBABTCHIEFF, Éditions ESKA, Paris

\section{Rédaction en chef}

Emmanuel DUBOIS DE PRISQUE, Chercheur associé, Institut Thomas More, Paris

Jean-Yves HEURTEBISE, Maitre de conférences, Université Catholique de Fujen, Taiwan, Chercheur Associé, CEFC, Hong-Kong

\section{Comité éditorial}

Xavier AUREGAN, Ingénieur de recherche, Mobis, NEOMAS BS, France

Daniel BELL, Doyen et professeur, Université du Shandong, Chine

Jean-Pierre CABESTAN, Directeur, Département de science politique et d'études internationales, Université Baptiste de Hong Kong

Jean ESMEIN, Historien et écrivain, docteur d'État es-lettres (études extrême-orientales), Senior Research Fellow de l'INSEAD

Qian HE, Sciences Po - Paris, France

Marie HOLZMAN, Sinologue, présidente de l'Association solidarité Chine

Michel JAN, Sinologue, chercheur Asie21-Futuribles

Zhe JI, Professeur, Institut national des langues et civilisations orientales, France

Léon LAULUSA, Professeur, Doyen pour les affaires académiques, ESCP Europe

Jean-Paul MARÉCHAL, Maître de conférences (HDR), Universitē Paris-Sud, chercheur au Collège d'études interdisciplinaires

Thierry PAIRAULT, Socio-économiste et sinologue, directeur de recherche émérite au CNRS (Centre Chine de l'EHESS, UMR 8173) et spécialiste des relations sino-africaines, France

Marianne PÉRON-DOISE, Chargée du programme Sécurité Maritime internationale, Institut de recherche stratégique de l'école militaire (IRSEM), France

Jean-Paul TCHANG, Économiste, co-fondateur de la Lettre de Chine

Benoît de TRÉGLODÉ, Directeur de recherche, Institut de recherche stratégique de l'école militaire (IRSEM), France

\section{Fabrication}




\section{TAÏWAN DES ANNÉES 1930 AUX ANNÉES 1950 : LA NAISSANCE D'UNE SOCIÉTÉ PLURALISTE}

\section{ont collaboré à ce numéro}

Philippe Chevalerias, Maître de conférences, Centre d'études en civilisations, langues et lettres étrangères (CECILLE), Université de Lille, Villeneuve d'Ascq, France

Jean-Christophe Defraigne est professeur à l'Institut d'études européennes de l'Université SaintLouis à Bruxelles et professeur invité à la Louvain School of Management

Emmanuel Dubois de Prisque, Chercheur associé à l'Institut Thomas More, corédacteur en chef de la revue Monde chinois, nouvelle Asie

Jean-Yves Heurtebise, Maître de conférences, Université FuJen, Taïwan, corédacteur en chef de la revue Monde chinois, nouvelle Asie

Coraline Jortay, Aspirante F.R.S.-FNRS, Universitê Libre de Bruxelles (ULB), EASt, Philixte, Striges, Maison des Sciences Humaines de l'ULB, Bruxelles, Belgique

Marie Laureillard, Université Lumière-Lyon 2 \& Institut d'Asie Orientale
Chanyueh Liu, ATER au Département d'Etudes chinoises à l'Université Paul Valêry, membre associê de l'Institut de Recherche Intersite d'Etudes Culturelles (IRIEC - EA740) de l'Université Paul Valêry Montpellier 3 et du Centre d'Etudes Asie-Est à l'Université Libre de Bruxelles (EASt-ULB-MSH)

Chun-ya Liu, PhD. Candidate. Géographie-cités UMR 8504, Panthéon-Sorbonne University (Paris 1) ; C.R.I.A., Institut de Géographie, Paris

Thierry Pairault, sinologue et socio-économiste, est directeur de recherche émérite au CNRS membre du Centre d'études sur la Chine moderne et contemporaine (UMR 8173, CNRS/EHESS)

Hicham Rouibah est doctorant en socio-économie à l'Universitê Paris-Diderot, membre du CESSMAA (UMR 245) et allocataire du programme Arts de l'IRD Stolojan-Filipesco Vladimir, Universitē Paris VII, CESSMA

Ben $\mathrm{Xu}$, docteur de l'université de Fudan et du Saint Marie College en Californie

Les articles signés expriment la seule opinion de l'auteur et ne sauraient engager la responsabilité de la revue.

Tous droits de traduction, d'adaptation et de reproduction par tous procédés réservés pour tous pays.

La loi du 11 mars 1957 n'autorisant aux termes des alinéas 2 et 3 de l'article 41, d'une part, que des copies ou reproductions strictement réservées à l'usage privé du copiste et non destinées à une utilisation collective et, d'autre part, que les analyses et courtes citations dans un but d'exemple et d'illustrations, « toute représentation ou reproduction intégrale, ou partielle, faite sans le consentement de l'auteur ou de ses ayants droit ou ayants cause, est illicite » (alinéa $1^{\text {er }}$ de l'art. 40).

Cette représentation ou reproduction, par quelque procédé que ce soit, constituerait donc une contrefaçon sanctionnê par les articles 425 et suivants du Code pénal.

Il est interdit de reproduire intégralement ou partiellement le présent ouvrage sans autorisation de l'éditeur ou du Centre Français de Copyright, 6 bis, rue Gabriel Laumain, 75010 PARIS.

Monde Chinois Nouvelle Asie 58 • Revue trimestrielle

(C) Editions ESKA - 2019

ISSN : 1767-3755 • ISBN : 978-2-7472-2921-0 • CPPAP : 0217 K 90325

Légende image de couverture : Palais présidentiel de Taipei à l'ère de la domination japonaise

Crêdits photos de couverture et pages intêrieures : D.R.

Imprimé en France 


\title{
La peinture à Taïwan des années 1930 aux années 1950 : échos artistiques du contexte socio-politique
}

\author{
Par Marie LAUREILLARD*
}

\section{Résumé}

Entre les années 1930 et 1950, l'art taïwanais, en particulier la peinture, reflète l'évolution politique de l'île et le passage de la gouvernance japonaise à celle du parti nationaliste chinois sous influence américaine. On distingue à la période japonaise deux courants, la peinture à la gouache sur soie dite nihonga et la peinture à l'huile à l'occidentale de style impressionniste. Le lendemain de la seconde guerre mondiale voit l'essor d'un réalisme qui s'accompagne d'une dimension critique nouvelle, avant que la tragique affaire du 28 février 1947 n'affecte profondément le milieu artistique. Puis, avec le repli de Tchang Kai-chek dans l'île en 1949, la peinture à l'encre traditionnelle chinoise devient bientôt le symbole de la "Chine libre ». Taiwan va s'ouvrir peu à peu aux tendances internationales et interpréter à sa manière l'expressionnisme abstrait alors en vogue aux États-Unis selon une voie radicalement opposée au réalisme socialiste prôné par la Chine communiste. Ainsi les choix esthétiques de l'époque peuvent-ils largement s'expliquer à la lumière de l'environnement socio-politique.

Mots-clés : Art taïwanais moderne, peinture " nihonga », peinture impressionniste, peinture "guohua », expressionnisme abstrait.

\begin{abstract}
Between the 1930s and the 1950s, Taiwanese art, especially painting, reflects the changes in the political situation of the island and the shift from a Japanese government to the governance of the Chinese Nationalist Party under American influence. We can see two strands during the Japanese period, gouache painting on silk called "nihonga" and Western oil painting in an impressionist style. After the Second World War, a new critical realism emerges, before the tragical 28 February Incident of 1947 profoundly affects the artistic community. Then, after the withdrawal of Chiang Kai-shek into the island in 1949, Chinese traditional ink painting soon becomes a symbol for "free
\end{abstract}


China". Taiwan progressively opens to international trends and interprets in its own way abstract expressionism, which is in fashion at the time in the United States, in the very opposite direction of socialist realism promoted by communist China. Thus, the aesthetic choices of the period can largely be explained in light of the socio-political environment.

Keywords: Taiwanese modern art, "nihonga" painting, impressionist painting, "guohua" painting, abstract expressionism.

Entre les années 1930 et 1950, l'art taïwanais, en particulier la peinture, reflète de manière frappante le destin politique de l'île et le passage de la gouvernance japonaise à celle du parti nationaliste chinois sous influence américaine. Sans doute ce phênomène de reflet résulte-t-il d'une forme de conditionnement politique imputable à la nature autoritaire des régimes politiques successifs. L'évolution artistique que l'on constate alors est d'une importance au moins égale au tournant linguistique de la littérature, brusquement passée de la langue japonaise à la langue chinoise. La peinture des années 1930-1945, qu'elle soit de style oriental ou occidental, paraît imprégnée d'une certaine quiétude, d'une absence d'esprit de rébellion contre la puissance coloniale et d'une recherche de couleur locale. À partir de 1945 apparaît une tendance au réalisme, tandis que la touche impressionniste, déjà présente dans les années 1930, se maintient. Mais on assiste bientôt au grand retour de la peinture à l'encre traditionnelle chinoise. De sa fusion avec l'expressionnisme abstrait d'origine américaine naîtra un courant moderniste vers la fin des années 1950. C'est cette page de l'histoire de l'art taïwanais très marquée par les fluctuations de la politique culturelle que nous voudrions examiner ici, en tentant d'expliquer les choix esthétiques de l'époque à la lumière de l'environnement socio-politique.

\section{Une esthétique japonaise}

À Taiwan, qui n'a obtenu le rang de province qu'en 1885 et se trouve à l'écart de la culture chinoise dominante, c'est une peinture à l'encre traditionnelle plutôt conservatrice qui est pratiquée sous les Qing. Accordant une place prépondérante au genre « fleurs et oiseaux » et aux thèmes religieux, elle se caractérise par un style proche de celui de la province côtière du Fujian située à $180 \mathrm{~km}$ de là, avec laquelle l'île entretient des rapports étroits pour des raisons de proximité géographique et linguistique. Les collections de peintures chinoises de grands maîtres sont plutôt rares, voire inexistantes sur lî́le.

Après sa défaite face au Japon en 1895, au terme de la première guerre sino-japonaise de l'histoire, la Chine doit céder Taiwan, qui devient officiellement colonie japonaise. " Dépecée » par les 
puissances européennes, la Chine a perdu à l'orée du XX $X^{\text {e }}$ siècle sa position centrale en Asie orientale. Le Japon, fort de sa victoire contre la Chine en 1895, puis contre la Russie en 1904, nourrit alors l'ambition de devenir le nouveau leader de la région, s'estimant plus avancé, plus civilisé - en s'occidentalisant - que les pays voisins. L'art joue un grand rôle dans la stratégie de développement nipponne, surtout après le passage d'un gouvernement militaire de l'île à un gouvernement civil en 1919. Introduite au Japon depuis l'ère Meiji à des fins de modernisation, la culture européenne pénètre alors à Taiwan, où les écoles secondaires vont initier les élèves à la pratique du dessin, à la peinture sur le motif, à l'usage de la perspective linéaire et des pigments à l'huile et à l'aquarelle.

À partir de 1927, le gouvernement japonais institutionnalise l'Exposition d'art de Taiwan (Taiten 台展) avec pour objectif d'assimiler la population en guidant le développement artistique insulaire. Cette exposition est calquée sur le modèle des Salons en France et de l'Exposition d'art impériale (Teiten 帝展) ayant lieu chaque année au Japon. Sous l'êgide d'un jury constitué d'artistes japonais influents, elle représente une plateforme importante pour les artistes taïwanais et l'une des rares voies de reconnaissance possibles en l'absence d'école des beaux-arts insulaire. Elle propose aux candidats deux sections de peinture : peinture de style oriental (tôyôga 東洋畫) et peinture de style occidental (seiyôga 元洋畫). Deux prix majeurs sont attribués ainsi que de nombreuses mentions.

En réalité, la peinture dite tôyôga ne se distingue en rien de la nihonga 日本畫 (《peinture japonaise "), appellation visant à la distinguer du style occidental. Apparue à Kyôto au cours de l'ère Meiji, dans les années 1880, cette peinture à la gouache sur soie de style extrême-oriental, dont les pigments sont fixés par de la colle, est influencée par les techniques européennes de représentation de la perspective ou de la lumière. Le terme tôyôga qui la désigne, terme également employé par les colons japonais en Corée, vise à inclure la population locale dans une nouvelle entité géoculturelle. En même temps, la peinture à l'encre, assimilée à la culture chinoise, est écartée pour des raisons éminemment politiques, prétendument en raison de son style jugé démodé et trop éloigné de la réalité. Comme le souligne Jason C. Kuo, il s'agit de toute évidence d'imposer par ce biais un « capital culturel » au sens où l'entend Pierre Bourdieu, c'est-à-dire les ressources culturelles dont dispose un individu. Bourdieu démontre ainsi que «la domination n'a plus besoin de s'exercer de manière directe et personnelle lorsqu'elle est impliquée dans la possession des moyens (capital économique et culturel) de s'approprier les mécanismes du champ de production et le champ de la production culturelle $»^{1}$.

À la première exposition figurent quarante œuvres de tôyôga, dont trois seulement réalisées par des artistes taïwanais : Kuo Hsueh-Hu, Lin Yu-Shan et Chen Jin. En 1926, Chen Chin 陳進 (19071998) a été admise à l'école d'art des femmes de Tokyo. Lin Yu-Shan 林玉山 (1907-2004) a étudié à l'école de peinture Kawabata de Tokyo en 1929 et en 1935. Kuo Hsueh-Hu 郭雪湖 (1908-2012), quant à lui, s'est formé en autodidacte en copiant des livres d'art à la bibliothèque. Nés et éduqués pendant la période de gouvernance japonaise, ces trois artistes sont alors considérés comme de dignes reprêsentants des nouveaux sujets du souverain nippon (kômin 皇民).

Seule artiste femme à jouir d'un certain renom dans le Taiwan de l'époque, Chen Chin, issue d'une riche et êminente famille de Hsinchu, est appréciée pour son esthétique raffinée et pour la grâce des dames de la haute société qu'elle aime à représenter vêtues de robes chinoises qipao alors à la mode à Shanghai, soulignant ainsi sa propre identité. Le concert, où deux femmes sont figurées dans un style évocateur de celui du Japonais Kaburagi Kiyokata 鏑木清方(1878-1972), est sélectionné pour la prestigieuse Exposition d'art impériale (Teiten) de 1934. La facture est soignée, les couleurs 
Photo 1 : Chen Chin (1907-1998), L'Accordéon, 1935, gouache sur soie, $180 \times 170 \mathrm{~cm}$, Musée des beaux-arts de Taipei.

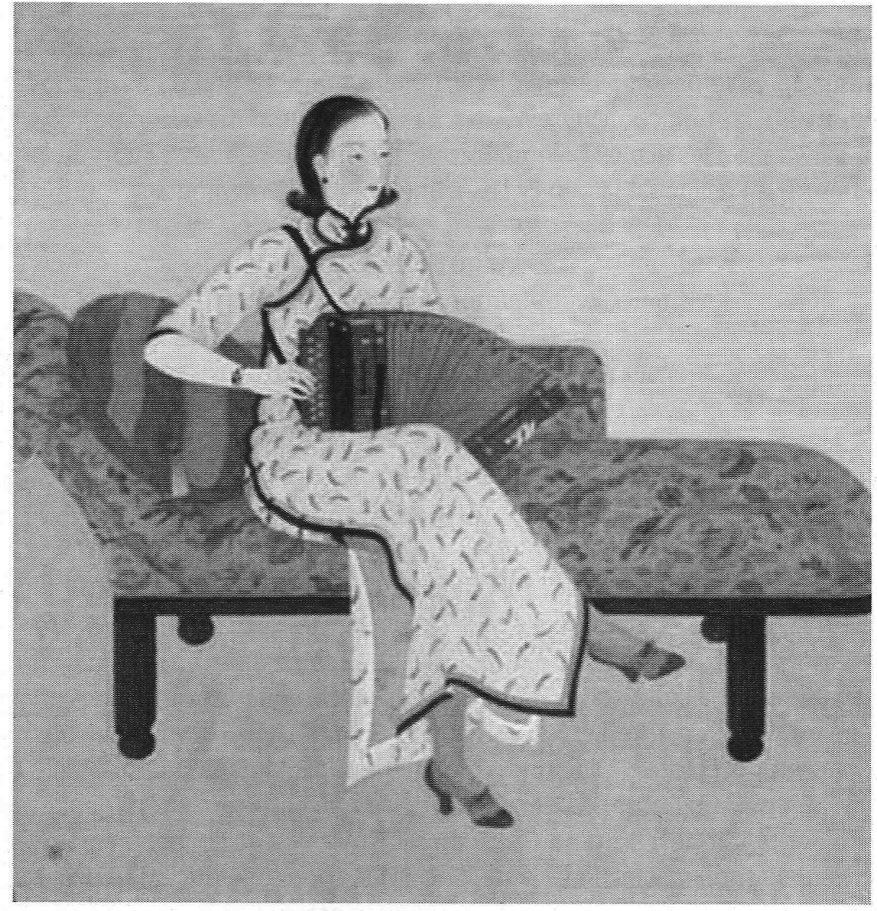

variées, les personnages sont représentés sur fond neutre avec une grande précision anatomique et un soin méticuleux apporté à leurs attributs (instruments de musique, bijoux...) et à leur environnement (meubles...), comme en témoigne également L'Accordéon, réalisé en 1935 [photo 1].

L'autre courant correspond à un style pleinairiste ou d'inspiration impressionniste, alors très en vogue au Japon, simplement qualifié de " peinture occidentale » ou seiyôga. Ce style, popularisé par Kuroda Seiki 黒田清輝 (1866-1924) qui avait étudié en France, s'intègre par conséquent tout naturellement à la politique japonaise d'assimilation. Le peintre Ishikawa Kin'ichirô 不川欽一郎 (1871-1945) contribue largement à introduire l'aquarelle et la peinture sur le motif lors de son séjour au collège de professeurs de Taipei de 1924 à 1932. Les peintres taïwanais adeptes du style « occidental » vont se former au Japon, comme Chen Cheng-Po 陳澄波 (1895-1947) et Liao ChiChun 廖繼春 (1902-1976), qui ont tous deux étudié à l'École des beaux-arts de Tokyo. Certains d'entre eux consolident leur apprentissage en Europe, comme Yen Shui-Long 顏水龍 (1903-1997) et Yang San-Lang 楊三郎 (1907-1995), qui séjournent en France au début des années 1930. Le premier, qui copie des œuvres du Titien et Ingres au Louvre et expose au Salon d'Automne de 1931, adopte un style réaliste aux couleurs chaudes et contrastées. Le second, avec qui il fondera la société Taiyang en 1934, participe quant à lui au Salon d'Automne de Paris en 1933. Il s'attachera plus tard à dépeindre les paysages urbains et ruraux de Taiwan avec une touche et une luminosité impressionnistes. 
Photo 2 : Liao Chi-chun (1902-1976), Cour de bananiers, huile sur toile, 1928, 129,2 × 95,8 cm, Musée des beaux-arts de Taipei.

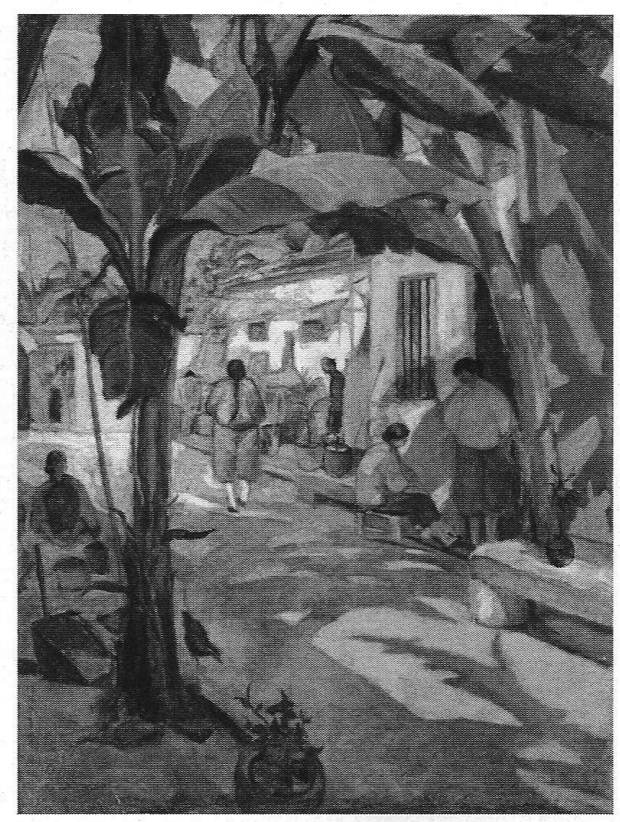

Qu'ils se rattachent à l'un ou à l'autre des deux courants, les artistes taïwanais sont encouragés à représenter les paysages locaux, censés révéler les progrès de la modernité introduite par les Japonais et satisfaire une soif d'exotisme envers une île qui apparait comme un petit paradis subtropical. La saveur régionale émanant des œuvres de Chen Cheng-Po, qui représente volontiers sa ville natale de Chiayi (Scène de rue en été, 1927) ou Liao Chi-Chun, qui excelle dans l'emploi de somptueuses couleurs (Cour de bananiers, 1928), sont particulièrement appréciées du public japonais [photo 2]. Dans le style tôyôga, Kuo Hsueh-Hu représente quant à lui un quartier commerçant prospère de Taipei parsemé d'enseignes, de bannières et d'affiches bariolées dans La rue du Sud en fête (1930). Cette œuvre frappe par l'atmosphère joyeuse, trépidante et animée qui se dégage de la profusion de couleurs et du savant assemblage d'inscriptions et de motifs décoratifs.

Ainsi les colons ont-ils su insuffler une vitalité culturelle nouvelle à une île engoncée dans des traditions éculées et amener les arts à évoluer à partir des années 1920. Certaines tentatives d'émancipation voient le jour parmi les insulaires : en novembre 1934 est fondêe la société d'art Taiyang, qui organise dix expositions indépendantes chaque année en mai jusqu'en 1944, afin de « faire valoir le caractère unique de la nation chinoise $»^{2}$ comme moyen de résister au contrôle japonais. Un courant éphémère du nom de " Mouve ", apparu en 1938, cherche également à prendre ses distances avec les normes en vigueur dans les expositions officielles. Il reprendra vie à travers la sociêté d'art Époque à partir de 1954. La société Taiyang, à laquelle adhèrent les principaux peintres occidentalistes de l'époque (Chen Cheng-Po, Li Mei-Shu 李梅樹, Li Shih-Chiao 李石樵, ainsi que Yang San-Lang et Yen Shui-Long) perdurera dans les années 1950. 


\section{La guerre et ses lendemains}

À partir de 1937, le Japon, en guerre contre la Chine, établit à nouveau un gouvernement militaire à Taiwan et durcit sa politique d'assimilation. L'étude du japonais est rendue obligatoire et les publications en chinois sont désormais interdites. Le contrôle des activités économiques, sociales et artistiques se resserre. En 1937, l'Exposition d'art de Taiwan est annulée à cause de l'entrée en guerre, avant que l'Exposition du Gouverneur (Futen 官展), sous la responsabilité directe du Bureau du Gouverneur, ne lui succède de 1938 à 1943.

Du jour au lendemain, en octobre 1945, après la défaite japonaise, Taiwan redevient province chinoise. L'usage du japonais est abandonné au profit de celui du mandarin. Yang San-Lang, avec le soutien d'autres peintres comme Chen Cheng-Po ou Chen Chin, suggère alors au gouvernement la tenue d'une exposition annuelle : la première Exposition provinciale de Taiwan (Shengzhan 省 展) est ainsi organisée dès octobre 1946 sur le modèle de la Taiten. Les artistes taïwanais établissent leurs propres instances d'évaluation. On garde au départ les concepts de peinture « orientale » et « occidentale » qui avaient prévalu durant la période de gouvernance japonaise. Cet événement jouera un rôle central pendant plusieurs décennies. Pour des raisons politiques évidentes, dès la première Exposition provinciale, la catégorie tôyôga est rebaptisée guohua. Dans cette catégorie nouvellement nommée guohua, les peintres taïwanais continuent à proposer des œuvres de style tôyôga/nihonga. Au début, la plupart des membres du jury (parmi lesquels Lin Yu-Shan, Chen Chin et Kuo Hsueh-Hu) pratiquent également ce style, qui est celui qu'ils connaissent le mieux.

Un nouveau courant prend bientôt son essor, axé sur un certain réalisme social. Hung Rui-Lin 洪 瑞麟 (1912-1996), peintre occidentalisant né à Taiwan et formé au Japon, décrit la vie des ouvriers et des mineurs, qu'il connaît bien pour avoir lui-même travaillé dans une mine de charbon. En quelques coups de pinceau sombres, il croque les mineurs, leur visage souffrant, leur musculature, suscitant l'empathie du spectateur [photo 3].

Ce courant caractérisé par un réalisme à la fois stylistique et thématique est également représenté par des artistes graveurs issus du Mouvement de la nouvelle gravure sur bois initié sur le continent par Lu Xun comme Chu Ming-Kang 朱鳴岡 (1915-2013) ou Huang Jung-Tsan 黃榮蹸 (1920-1952). Chu Ming-Kang, arrivé à Taiwan en 1946, décrit la vie des humbles, encore vêtus à la japonaise, et des sans-abris, révélant la dure réalité quotidienne du Taiwan de l'après-guerre. Il rejoindra Hong-Kong en 1948, puis la Chine.

Originaire de Chongqing au Sichuan, Huang Jung-Tsan a étê recruté comme enseignant à Taiwan dès 1945. Impliqué dans le mouvement de l'art moderne, attentif à la vie locale, il est célèbre pour avoir réalisé l'une des rares représentations visuelles de «l'Incident du 28 février 1947 », soulèvement populaire contre le gouvernement écrasé dans le sang par le Parti nationaliste chinois (Kuomintang) au pouvoir, alors dirigé depuis le continent par Tchang Kai-chek. Sa gravure, intitulée La terrible inspection (1947), lui vaudra d'être arrêté et emprisonné en décembre 1951 et d'être exécuté un an plus tard pour trahison, espionnage et propagande communiste. Ainsi les premières étincelles d'une critique sociale sont-elles rapidement éteintes.

De fait, les lendemains de la guerre apportent bien des déconvenues aux artistes taïwanais. Comme les écrivains, ils se voient marginalisés par la nouvelle bureaucratie culturelle. Ils subissent de plein fouet la répression politique de 1947 qui les marque profondément et compromet la liberté 
Photo 3 : Hung Jui-Lin (1912-1996), Rassemblement avant le travail, 1955, encre et aquarelle sur papier, $33 \times 22 \mathrm{~cm}$, Musée des beaux-arts de Taipei.

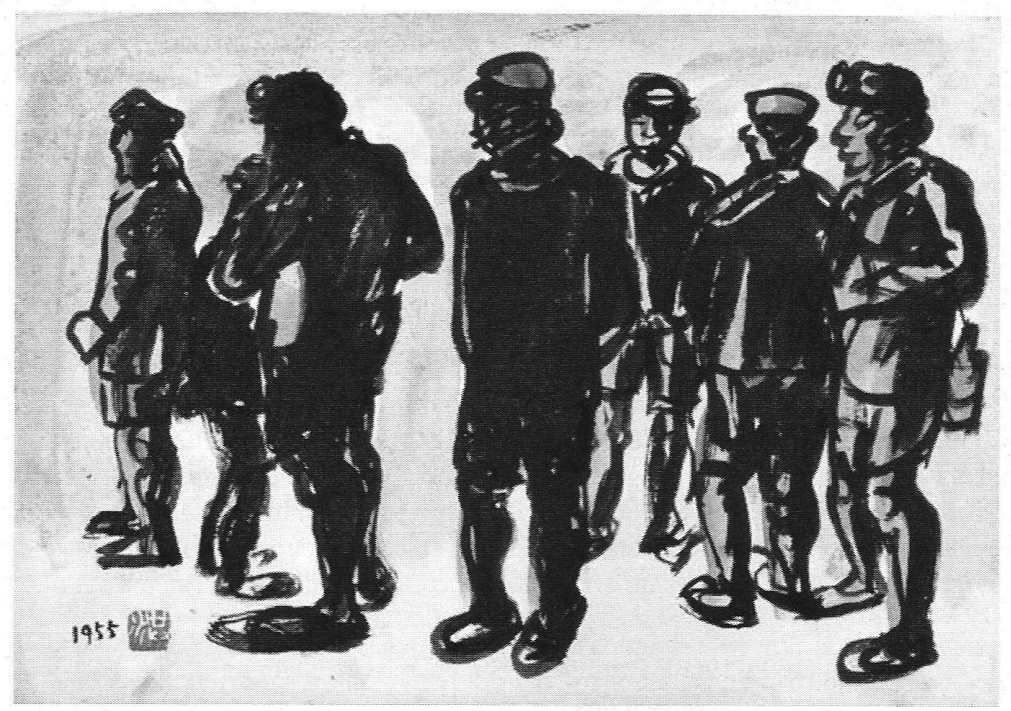

d'expression qu'ils avaient cru pouvoir obtenir en 1945. Le peintre Chen Cheng-Po comptera lui aussi parmi les victimes du tragique événement de 1947. Quelques mois plus tard, la loi martiale sera instaurée pour près de quarante ans et la "Terreur blanche » se répandra. Ainsi Yang SanLang décrit-il la morne atmosphère de l'après-guerre : « Toutes les activités culturelles de quelque importance stagnaient, tandis que la scène artistique tout entière restait morose et silencieuse. Les peintres autrefois actifs et dynamiques dans leurs activités artistiques se trouvaient dans une situation dêlicate ou optaient pour une retraite passive et solitaire. $»^{3}$

\section{Le retour de la peinture chinoise traditionnelle}

En 1950, dans le sillage de Tchang Kai-chek, deux millions de Chinois du continent se réfugient à Taiwan, alors peuplé de seize millions d'autochtones. La peinture chinoise traditionnelle va rapidement devenir le symbole de la "Chine libre », qui met l'accent sur un savoir prétendument " orthodoxe » et sur la morale confucéenne. Les peintres Pu Hsin-Yu et Huang Chun-Pi incarnent le lien avec le continent et jouissent du patronage de fonctionnaires de haut rang du gouvernement nationaliste.

Né à Pékin, Pu Hsin-Yu 溥心蕃 (1896-1963), cousin du dernier empereur Puyi, a assisté à l'effondrement de la cour impériale. Il s'est plongé dans l'étude des classiques et de la poésie, devenant un poète respecté et exprimant ses sentiments à travers la calligraphie et la peinture. Fermement opposé à la coopération de Puyi avec les Japonais, il suit les nationalistes à Taiwan, où il est nommé professeur d'art à l'Université Normale Nationale de Taiwan. Très estimé et honoré, ce peintre à la touche élégante et méticuleuse, qui a eu accès à la collection impériale de par son origine familiale, 
a étê très marqué par les paysages dēlicats des Song ou par certains peintres Ming, comme Tang Yin 唐寅 (1470-1524) [photo 4]. Affectant une attitude détachée, il est de plus en plus fasciné par le thème du fantôme qui exprime peut-être sous son pinceau un certain sentiment d'exil. C'est sous son influence que Lin Yu-shan se tournera vers la peinture chinoise traditionnelle.

Autre peintre tenu en haute estime, Huang Chun-Pi 黃君璧 (1898-1992), attaché à l'étude d'après nature, révèle une tendance naturaliste alors que sa méthode d'enseignement repose largement sur la copie. Certains effets de lumière laissent entrevoir une influence occidentale, voire japonaise, quand lui-même se réclame d'une orthodoxie chinoise ${ }^{4}$. Chiang Chao-Shen 江兆申 (1925-1996) sera l'un de ses disciples les plus doués.

Le gouvernement est ainsi persuadé que les valeurs traditionnelles doivent être préservées face à la menace communiste. En fuyant la Chine, le Kuomintang a apporté la partie la plus précieuse de la collection d'art impériale, considérée comme une sorte de régalia. Dans l'attente de la reconquête du continent, en laquelle Tchang Kai-chek croira longtemps, un immense musée sera bâti à Taipei pour accueillir la collection en 1965. Conforté dans sa légitimité par ce demi-million d'objets si précieux, le gouvernement nationaliste se considère comme le gardien de l'héritage chinois, qu'il érige en dogme. Le choix d'honorer un art considéré comme traditionnel (même s'il n'existe pas,

Photo 4 : Pu Hsin-Yu (1896-1963), Paysage, 1959, encre et couleur sur papier, 65,8 $\times 27,8 \mathrm{~cm}$, Musée des beaux-arts de Taipei.

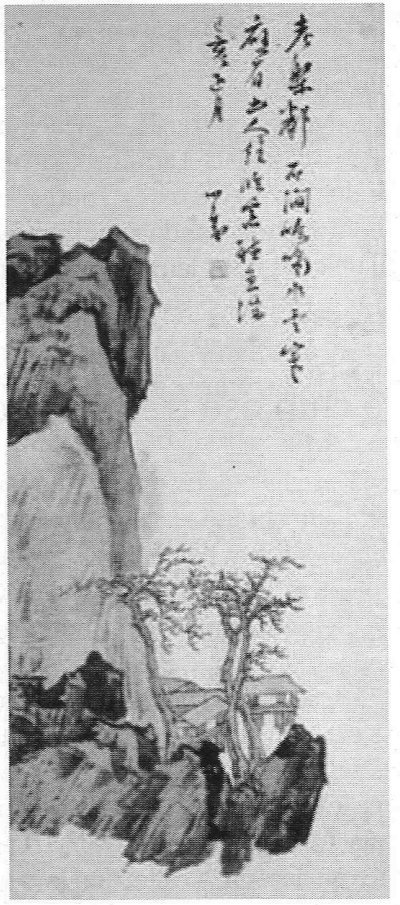


à l'évidence, d'art «pur » ou « authentique $»^{5}$ ) s'oppose en particulier à celui du continent, qui cherche à en détruire les vestiges en préconisant le style réaliste socialiste. Le terme guohua, né de la confrontation avec les puissances occidentales (comme le terme nihonga au Japon, d'ailleurs), revêt précisêment une forte connotation nationaliste. La politique culturelle sera essentiellement marquée par le projet, toujours plus irréaliste, de « libérer » la Chine.

La peinture à l'encre de style chinois va ainsi devenir le courant majeur, soutenu par le pouvoir politique, qui l'assimile à l'orthodoxie culturelle au détriment de la peinture de style japonais. Les exigences du Kuomintang en matière d'art rappellent indéniablement celles du gouvernement colonial japonais par une évidente similarité structurelle : la persistance de la division du monde entre art « d'Occident » et " d'Orient » et le refus d'hybridité sont frappants. Cette continuité semble au moins aussi importante que la discontinuité entre styles prônés par l'empire du Japon et par le Kuomintang de la loi martiale. N'y a-t-il pas là un commun refus de la possibilité de mélange ?

Huang Chun-Pi, Pu Hsin-Yu et Ma Shou-Hua 馬壽華 intègrent le jury vers 1950 : formés à la peinture lettrée et à la calligraphie, ils copient les anciens maîtres, dépeignent rarement leur expérience quotidienne et s'interressent davantage aux paysages de la Chine continentale qu'à l'environnement taïwanais. Pu Hsin-Yu et Huang Chun-Pi connaissent un grand succès en tant que professeurs de peinture traditionnelle, le second ayant pour élève Madame Tchang Kai-chek. Au début des années 1950, une " controverse sur l'orthodoxie de la peinture chinoise » va voir le jour dans les milieux artistiques, illustrant les tensions résultant du passage de cinquante ans de colonisation japonaise à la gouvernance du Kuomintang ${ }^{6}$. Animés de sentiments anti-japonais, les adeptes de la guohua ou « peinture chinoise » de style traditionnel, réfugiés du continent à la suite de Tchang Kai-shek, n'admettent pas la moindre allégeance au style japonisant dit tôyôga ou nihonga évoqué plus haut. Pu Hsin-Yu critique ainsi l'usage des techniques « occidentales » qu'il y décēle et appelle à se fonder davantage sur l'art de la calligraphie. Liu Shih 劉獅 (1910-1997), neveu du célèbre peintre Liu Haisu 劉海蒌, dénigre ouvertement la nihonga qu'il juge tout juste bonne pour la décoration, manquant cruellement de la « résonance du souffle » (qiyun 氣韻) que l'on prête d'ordinaire à la peinture à l'encre chinoise? Les tenants de ce style se défendent en déclarant que l'on ne peut réduire la peinture taïwanaise à de la nihonga du fait de la saveur régionale et du réalisme qui l'imprègnent.

Les artistes continentaux d'obédience « orthodoxe » épousent la vision politique des autoritês, qui, de manière générale, se méfient de l'empreinte japonaise encore si perceptible chez les insulaires. En refusant catégoriquement de considérer le style de la tôyôga ou nihonga comme une variante de la guohua, ils êmettent un jugement presque « racialiste », qui fait songer à une division sociale, voire ethnique, prolongeant d'une certaine manière celle qui existait avant 1945 entre Japonais et Taïwanais. La controverse se poursuivra jusqu'à la fin des années 1950, la tôyôga tombant de plus en plus en disgrâce. Après une longue période de silence, celle-ci sera rebaptisée dans les années 1980 jiaocaihua 膠彩畫 ( ( peinture aux pigments et à la colle »), appellation mettant l'accent sur les matériaux utilisés plutôt que sur une quelconque appartenance géopolitique.

Attaquant souvent les traditionalistes dans sa volonté de s'établir lui-même comme chef de file de la peinture moderniste, Liu Kuo-Sung 劉國松 (1932-) s'en prend également à plusieurs reprises au style japonisant de certains artistes, qu'il considère comme non chinois. Il écrit en 1954 un article intitulé "La peinture nihonga n'est pas de la peinture guohua », allant même jusqu'à accuser le "groupe qui étrangle la culture nationale et attaque l'art de la quintessence chinoise » en 1959 dans une lettre ouverte au ministre de la culture ${ }^{8}$. 


\section{L'ouverture à l'influence américaine et au monde}

À partir de 1950, la guerre de Corée fait de Taiwan un avant-poste américain majeur dans le Pacifique et se traduit par un soutien fort, à la fois militaire, financier et économique de la part des États-Unis au régime de Tchang Kai-chek. Le Kuomintang va reprendre à son compte le concept américain de sauvegarde du «monde libre ». Bien que la culture ne soit pas l'une des préoccupations premiēres du gouvernement, une école nationale des beaux-arts, la première du genre, est fondée en 1955 à Taiwan.

À la différence de la peinture nihonga, la peinture à l'huile de type impressionniste, qui avait été introduite à Taiwan par les Japonais, ne suscite aucune hostilitê, car elle répond à une position pro-occidentale et anticommuniste. Sur le plan esthétique, Liu Kuo-Sung, le chef de file de la sociétê de Mai qui cherche ardemment à innover, lui reprochera pourtant de rester enfermée dans un même style sans chercher à aller au-delà̄.

Dès 1950, les bases du futur mouvement moderniste de Taiwan sont jetées par la revue Art nouveau, sous l'égide de l'artiste Ho Tie-Hua 何鐵華 (1910-1982), qui présente les divers courants artistiques occidentaux postérieurs au cubisme et au fauvisme. À la différence de la Chine à la même époque, Taiwan autorise une libre circulation de ses ressortissants dans les divers pays du monde et encourage les artistes à partir étudier à l'étranger. Le mouvement moderniste apparaît dans un premier temps comme une menace envers l'hégémonie culturelle du Kuomintang, qui finit pourtant par le tolêrer. Si l'esprit critique ne peut alors se manifester sur le plan idéologique, il peut tout de même s'exprimer dans le domaine esthétique.

Comme le souligne le spécialiste Hsiao Chong-Ray, l'essor de l'expressionnisme abstrait à Taiwan peut être, dans une certaine mesure, attribué à la politique d'affrontement de la Guerre froide, qui préconise de suivre une voie radicalement opposée au réalisme socialiste promu par la Chine communiste, mais il prolonge en réalité une réflexion plus ancienne sur une possible réforme de la peinture chinoise traditionnelle ${ }^{10}$. De plus en plus, les artistes taïwanais cherchent à s'inscrire dans une modernité internationale, estimant que la peinture traditionnelle, figée dans le conformisme, demande à être revivifiée. Tout aussi insatisfaits de l'immobilisme de la peinture à l'huile, ils se tournent vers les dernières tendances mondiales.

La société de la Cinquième Lune (Wu yue 五月), fondée en mai 1957 à l'instigation de Liao ChiChun, apporte ainsi un souffle nouveau au monde de l'art en proposant une synthèse entre art traditionnel chinois et expressionnisme abstrait. Le libre tracé du pinceau et les figures abstraites de ce dernier évoquent la calligraphie chinoise. Sur le modèle du Salon de Mai, célèbre manifestation parisienne, ils choisissent comme nom de groupe « Wu yue », qui signifie " mois de mai », qu'ils angliciseront par la suite en « Fifth Moon».

Liu Kuo-Sung (1932-) et Chuang Che 莊喆 (1934-), tous deux d'origine continentale, vont réinterpréter la linéaritê picturale et la calligraphie, le rôle du vide ainsi que certaines approches atmosphériques propres à la peinture chinoise ancienne en s'orientant complètement vers l'abstraction à partir de 1959. Chuang Che, qui a grandi au sein d'une famille de lettrés, est le fils d'un calligraphe de renom et vice-président du musée de la Cité interdite, qui, en 1948, participe activement au convoyage des œuvres de Pékin à Taipei. Alors âgé de treize ans, le futur peintre, qui assiste à cette formidable épopée, restera toujours admiratif de la peinture traditionnelle chinoise. 
Photo 5 : Liu Kuo-Sung (1932-), Flux rythmique, encre sur papier, 134,8 × 77,5 cm, Musée des beaux-arts de Taipei.

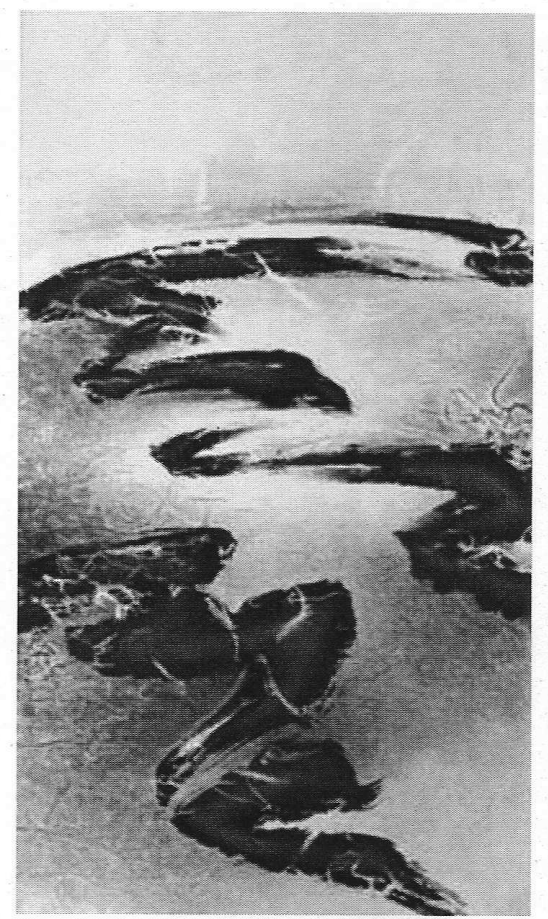

Le médium importe pourtant moins à ses yeux qu'à ceux de Liu Kuo-Sung. Celui-ci, théoricien du groupe, se tourne d'abord vers la peinture à l'huile, fasciné par l'œuvre de Cézanne, pour prôner ensuite la reconquête de la tradition chinoise et le retour à l'usage de l'encre sur papier. Pour lui, l'abstraction est signe de modernité et la peinture à l'encre incarne véritablement la Chine [photo 5].

En novembre 1957, c'est au tour de la société de l'Orient (Ton-Fan 東方), sous l'impulsion de Li Chun-Shan 李仲生 (1912-1984), d'organiser une exposition qui vise également à remettre l'art traditionnel chinois au goût du jour en intégrant certains apports occidentaux. Afin de détourner les soupçons envers la nature politique de ce nouveau courant, accusé d'être communiste parce que s'écartant de la ligne prônée par le Kuomintang, on y associe certains artistes espagnols. Surnommé le "mentor de l'art moderniste de Taiwan », Li Chun-Shan, originaire du Guangdong, a étudié au Japon dans les années 1930 et enseigné à l'École des beaux-arts de Hangzhou. Dans son atelier de Taipei, entre 1951 et 1956, il dispense un enseignement anti-académique, appelant à éveiller son propre potentiel créatif plutôt que de copier des modèles de plâtre, recourant ainsi à une méthode jugêe révolutionnaire dans un environnement très conservateur. Quant à Hsiao Chin 蕭勤 (1935-), qui s'installe en Europe dès 1956 et puise à la source du zen, du tantrisme et de la calligraphie pour réaliser des compositions à l'huile abstraites et géométriques aux vibrantes couleurs, il participe activement à ce mouvement, qu'il contribue largement à faire connaître à l'étranger. 
Bien qu'intégrant un langage artistique occidental, les expérimentations de tous ces jeunes peintres sont loin d'être une copie de l'art abstrait américain. Un cadre conceptuel est élaboré avec le soutien d'intellectuels tels que le poète Yu Kwang-chung 余光中 afin d'incorporer l'abstraction aux traditions autochtones. L'essor du modernisme à Taiwan est également favorisé par certains facteurs externes : en 1956, des œuvres d'artistes taïwanais sont sélectionnées à la Biennale internationale de Sao Paulo, attestant la reconnaissance internationale dont font l'objet les modernistes « chinois ».

Ainsi, la peinture chinoise traditionnelle se trouve ressourcée grâce à une fusion entre Chine et Occident et parvient à des résultats inédits tout en se dégageant des entraves de l'art figuratif. Dans la même veine, une société de Gravure sur bois moderne privilégiant l'abstraction, représentée par des artistes comme Chen Ting-Shih 陳庭詩 (1916-2002) ou Liao Shiou-Ping 廖修本(1936-), voit le jour en 1959.

Ce bref parcours nous permet ainsi de mesurer à quel point les courants artistiques ont été étroitement dépendants de la situation politique à Taiwan entre les années 1930 et les années 1950. Alors qu'à la période de gouvernance japonaise se distinguent deux courants, la peinture « occidentale " et la peinture "orientale", on retrouve une division comparable après la prise du pouvoir par le Kuomintang en 1945, à ceci près que l'on cherche à s'affranchir de toute influence japonaise au profit d'un style pictural considéré comme authentiquement chinois. Pendant toute cette période, les aspects politiques et artistiques se mêlent si étroitement que la création artistique peut être considérée à la fois sous l'angle du pouvoir (elle se met au service de la politique) et de l'artiste (qui affirme une position politique à travers ses œuvres). On peut se demander si, dans leur volonté de se mesurer aux Japonais ou de se conformer aux nouvelles directives du pouvoir chinois, les artistes taïwanais n'ont pas dû renoncer d'une certaine manière à une part de leur identité culturelle. Un artiste comme Lin Yu-Shan (1907-2004), formé au Japon à l'école d'art Kawabata en 1926, où la découverte d'anciennes peintures chinoises de grands maîtres l'avait dêcidé à étudier la peinture à l'encre plutôt que la peinture à l'huile, est emblématique de cette évolution : il se conforme aux changements de goûts esthétiques, passant avec aisance du raffinement de la tôyôga au trait enlevé de la guohua après 1949, puis à l'abstraction vers 1960, ce qui peut apparaître comme la marque d'un certain opportunisme, mais également d'une faculté d'adaptation et de dons artistiques remarquables [photo 6]. Cependant, bien que la politique culturelle ait largement contribué à orienter les choix esthétiques, malgré les contraintes pesant sur la créativité des artistes, celle-ci n'a jamais été étouffée, aussi bien du temps de la colonisation japonaise qu'à l'époque du régime autoritaire du Kuomintang. Après 1950, les arts n'ont jamais été aussi uniformisés que sur le continent sous le contrôle de Mao, où l'on prône ouvertement le style réaliste socialiste d'influence soviétique et où l'on tolère une peinture à l'encre assujettie à l'idéologie communiste. Plus ou moins libres dans leurs choix esthétiques, les artistes taïwanais ont pu s'ouvrir à de multiples influences, et les tensions politiques n'ont pas empêchê le dêveloppement d'un art riche et variê des annểes 1930 aux années 1950, formant le socle du développement ultérieur de l'art taïwanais, qui, avec l'émancipation des milieux artistiques à l'égard du pouvoir vers 1960, allait s'engager dans une voie originale et prendre peu à peu conscience de sa valeur et de sa spécificité. 
Photo 6 : Lin Yu-Shan (1907-2004), Tigre rôdeur, 1972, encre et couleur sur papier, $61 \times 74 \mathrm{~cm}$, Musée des beaux-arts de Taipei.

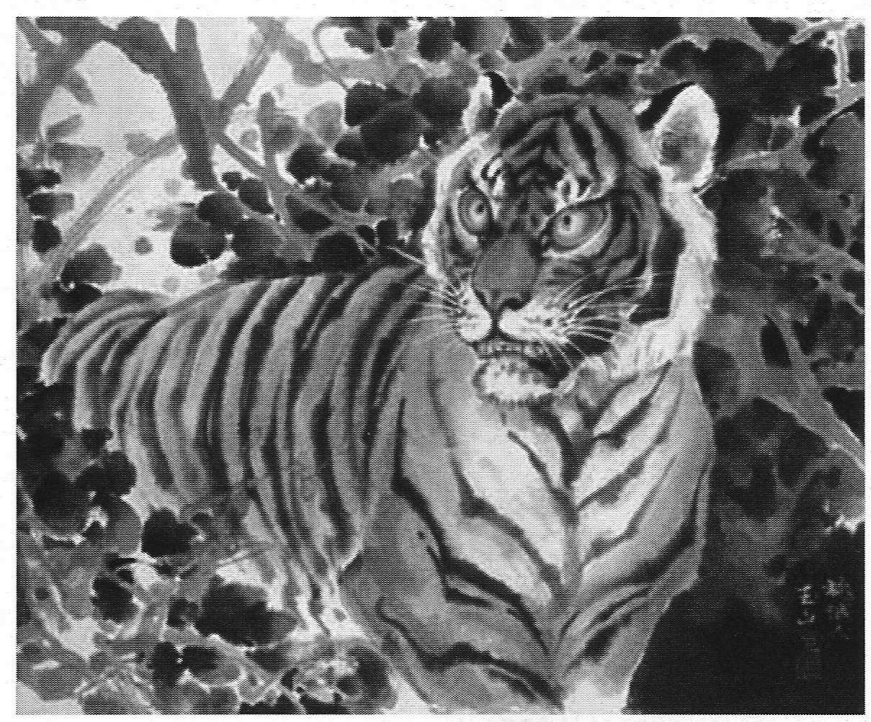

\section{Notes}

* Université Lumière-Lyon 2 \& Institut d'Asie Orientale.

${ }^{1}$ Pierre Bourdieu, Esquisse d'une théorie de la pratique, Paris : Droz, 1972, p. 183, cité par Jason C. Kuo dans Art and Cultural Politics in Postwar Taiwan, Taipei; SMC Publishing Inc; London and Seattle: University of Washington Press, 2000, p. 37.

${ }^{2}$ Lü Peng, Histoire de l'art chinois au XXe siècle, Paris : Somogy, 2013, p. 178

${ }^{3}$ Lü Peng, Histoire de l'art chinois au XXe siècle, op. cit., p. 442.

${ }^{4}$ Jason C. Kuo, Art and Cultural Politics in Postwar Taiwan, Taipei: SMC Publishing In; Seattle and London: University of Washington Press, 2000, p. 78.

${ }^{5}$ Jason C. Kuo, Art and Cultural Politics in Postwar Taiwan, op. cit., p. 78.

${ }^{6}$ Jason C. Kuo, Art and Cultural Politics in Postwar Taiwan, op. cit., p. 61.

${ }^{7}$ Jason C. Kuo, Art and Cultural Politics in Postwar Taiwan, op. cit., p. 75.

${ }^{8}$ Lü Peng, Histoire de l'art chinois au XX $X^{e}$ siècle, op. cit., p 444 et p. 446.

${ }^{9}$ Lü Peng, Histoire de l'art chinois au XXe siècle, op. cit., p. 446.

${ }^{10}$ Sabine Vazieux (êd.), From China to Taiwan : les pionniers de l'abstraction / Pioneers of Abstraction, Hsiao ChongRay, "Brève histoire de la peinture abstraite à Taiwan ", Bruxelles : Éditions Racine-Lannoo, 2017, p. 23. 